\title{
Development of compact highly sensitive beam position monitors for storage rings
}

\author{
Falastine Abusaif
}

Received: date / Accepted: date

\begin{abstract}
The history of the search for electric dipole moment (EDM)takes us back in time for more than five decades. EDMs have gained interest due to the fact that they violate the $C P$ symmetry. Hence, they potentially provide additional sources of $C P$ violation and possibly contribute to the resolution of one of the greatest puzzles of cosmology; mainly to explain the matter abundance in the universe.

The JEDI collaboration is currently preparing for measuring the Deuteron EDM in the COoler SYnchrotron (COSY). One of the major challenges is the precise knowledge about the beam position along the storage ring. Transverse beam positions largely control the systematic errors. Thus the development of compact and highly sensitive Beam Position Monitors (BPMs) is particularly important for precision experiment like the EDM searches. This paper describes the development of the latest set of Rogowski coils as compact beam position monitors. It also describes some of the preliminary results towards optimizing the sensitivity of these coils by controlling the noise sources and approaching improved signal to noise ratio (SNR).
\end{abstract}

Keywords Beam Position Monitors $\cdot$ Rogowski coil $\cdot$ SNR $\cdot$ Storage rings

\section{Introduction}

The Rogwoski pick-up coil used as a BPM is made mainly from a toroidal coil segmented into four seperate quadrants (see Fig 1). A time-varying current along the longitudinal axis of this coil will result in the formation of four induced voltage signals, thanks to Faraday's law of electromagnetic induction [1]. For these monitors to be able to deliver beam positions, a quantity called the difference over the sum (see

Falastine Abusaif

Forschungszentrum Jülich

Telefon: +49 $246161-2147$

Fax: +49 2461 61-3930

E-mail: f.abusaif@fz-juelich.de 
equations 1 and 2) is calculated from the individual voltage signals [2].

$$
\begin{aligned}
& \frac{\Delta U_{x}}{\Sigma U_{i}}=c_{1} x-c_{2}\left(x^{3}-3 y^{2} x\right)+c_{3}\left(x^{5}-10 y^{2} x^{3}+5 y^{4} x\right), \Delta U_{x}=U_{1}+U_{2}-U_{3}-U_{4} \\
& \frac{\Delta U_{y}}{\Sigma U_{i}}=c_{1} y-c_{2}\left(y^{3}-3 x^{2} y\right)+c_{3}\left(y^{5}-10 y^{3} x^{2}+5 y x^{4}\right), \Delta U_{y}=U_{1}+U_{4}-U_{2}-U_{3}
\end{aligned}
$$

Where $c_{i}$ are constants that depend only on the coil parameters as the inner and outer radii. $U_{i}$ is the induced voltage on the $i$ th quadrant, $\mathrm{x}$ and $\mathrm{y}$ are the transverse beam position coordinates. Figure 2 shows a theoretically predicted grid map of the horizontal and vertical ratios for different current positions in a range of $\pm 12 \mathrm{~mm}$.

\section{Beam time test}

After construction and calibration tests in the lab, the Rogwoski coil BPMs were installed in the COSY storage ring. Fig 3 shows the positions of the beam as delivered from one of the Rogowski coil BPMs during the beam time run of January/February 2018.

\section{Noise measurement}

As a part of the present optimization for these BPMs, a noise test was carried in the lab using the lock-in amplifier (which is the device used to measure the induced

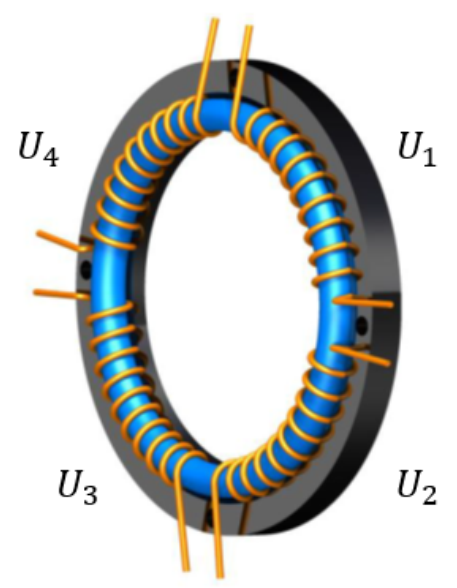

Fig. 1: Schematic diagram for the Rogowski coil with induced voltages on four quadrants 


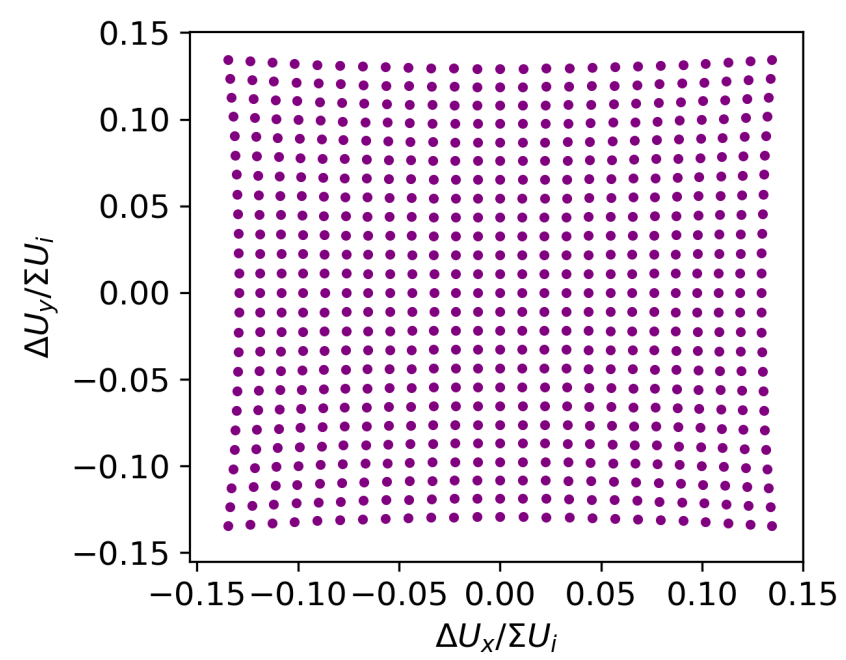

Fig. 2: Theoretical prediction of horizontal and vertical ratios for beam current transverse positions uniformly changing in a range of $\pm 12 \mathrm{~mm}$

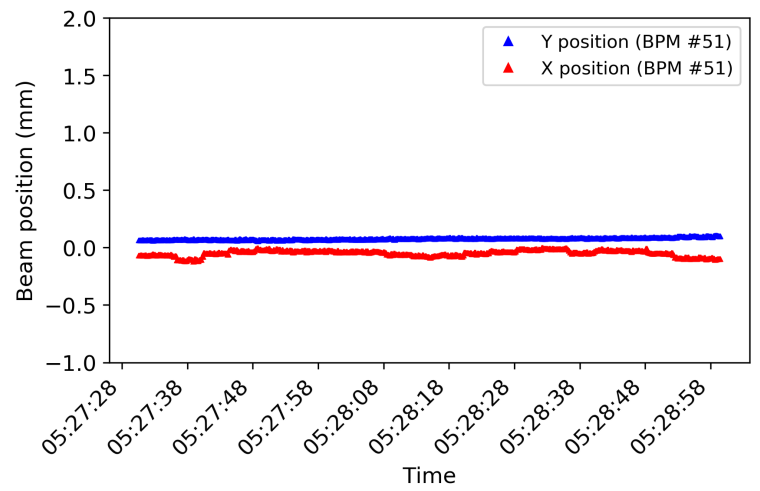

Fig. 3: Transverse beam positions (red and blue) during some cycle of January/February beam time 2018 with the clear effect of some 4-seconds orbit control operation on the horizontal beam bosition 
voltage at the reference frequency of $750 \mathrm{kHz}$ ) and another two versions of signal preamplifiers. The test was performed at different device settings to inspect the noise level in the measurement. One of the preliminary results was that a higher voltage range at the input will result in more noise level (see fig 4). For the frequency of interest (750 kHz-beam revolution frequency), the measured noise spectral density in $\mathrm{nV} / \sqrt{\mathrm{Hz}}$ was 42.33 and 2.92 for the voltage ranges of $1 \mathrm{~V}$ and $1 \mathrm{mV}$, respectively.

\subsection{Signal to noise ratio}

The signal to noise ratio for a beam current of $1 \mathrm{~mA}$ was measured from one quadrant of the coil at different filter settings. This filter is an internal low pass one in the circuit-design of the lock-in amplifier. Table 1 shows the settings and the results. As

Table 1: Average SNR measured from one quadrant of the BPM at the current strength of $1 \mathrm{~mA}$ for the fourth and eighth order filter settings

\begin{tabular}{c|cc}
\hline Filter slope & $24 \mathrm{~dB} / \mathrm{Oct}$ & $48 \mathrm{~dB} / \mathrm{Oct}$ \\
$3 \mathrm{~dB}_{\text {cut }- \text { off }}$ frequency & $6.81 \mathrm{~Hz}$ & $1 \mathrm{mHz}$ \\
Average SNR & $1.36 * 10^{3}$ & $1.9 * 10^{6}$ \\
\hline
\end{tabular}

shown in the table, an SNR could be improved by a factor of thousand if the eighth order filter with $1 \mathrm{mHz}$ cut-off frequency was used in the measurement. Unfortunately, this setting corresponds to a time constant of $48 \mathrm{~s}$, which is impractical for fastresponse detection of any potential beam changes in the synchrotron.

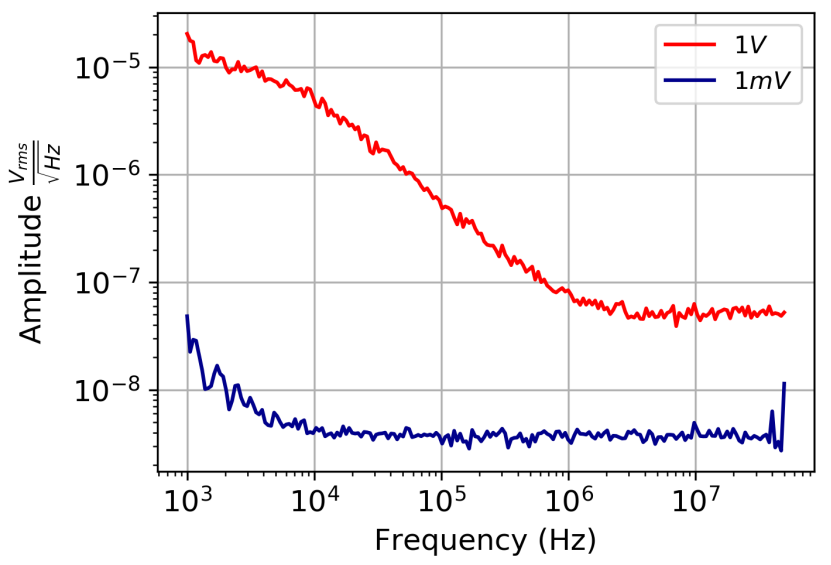

Fig. 4: Noise spectrum at the two input range settings $1 \mathrm{mV}$ and $1 \mathrm{~V}$ in the lock-in amplifier 


\section{Conclusion}

The development of the Rogowski coil-based BPM is one important step in the future of accelerator physics, not only can these monitors be used in EDM searches, on contrast, they can be widely exploited in distinct experimental fields, and this is mainly due to their high sensitivity and compact design of less than $10 \mathrm{~cm}$.

\section{References}

1. Trinkel, F.:Development of a Rogowski coil Beam Position Monitor for Electric Dipole Moment measurements at storage rings. https://d-nb.info/1162846038/34 (2017). Accessed 30 July 2018.

2. Bugg, D. V.: Electronics: circuits, amplifiers and gates, 85-86. CRC Press (2006) 